Si ngl e Radi al /Azi muthal Node Phot oni c Crystal Fi ber S Wth Ani sot ropi C El I i pti cal-Hol e Lattice core

\begin{tabular}{|l|l|}
\hline 著者 & ZHENG Zhong, TSUJ I Yasuhi de, EGUCH Nasashi \\
\hline $\begin{array}{l}\text { j our nal or } \\
\text { publ i cat i on t i t l e }\end{array}$ & I EEE Phot oni cs Technol ogy Let ter s \\
\hline vol une & 29 \\
\hline number & 16 \\
\hline page r ange & $1285-1288$ \\
\hline year & 2017- 08-15 \\
\hline URL & ht t p: //hdl . handl e. net /10258/00009484 \\
\hline
\end{tabular}




\title{
Single Radial/Azimuthal Mode Photonic Crystal Fibers With Anisotropic Elliptical-Hole Lattice Core
}

\author{
Zheng Zhong, Student Member, IEEE, Yasuhide Tsuji, Member, IEEE, and Masashi Eguchi, Member, IEEE
}

\begin{abstract}
Novel single radial/azimuthal mode photonic crystal fibers (PCFs) with an anisotropic elliptical-hole lattice core (EHLC) are proposed and demonstrated numerically. High birefringence is achieved by introducing elliptical holes into the core, which is considered as an effective way to break the symmetry. With a full vectorial finite element method, we can optimize the fiber parameters and also investigate the wave guiding characteristics in detail. A bandwidth of $210 \mathrm{~nm}$ (the wavelength range from 1.45 to $1.66 \mu \mathrm{m}$ ) has been achieved for single radial mode transmission by the PCF with an actinomorphic EHLC. Meanwhile, a bandwidth of $100 \mathbf{~ n m}$ (the wavelength range from 1.50 to $1.60 \mu \mathrm{m}$ ) for single azimuthal mode transmission has been also achieved by the PCF with a multi-annulus EHLC. It is anticipated that the proposed PCFs would be of very much convenience in mode multiplexing systems.
\end{abstract}

Index Terms-Photonic crystal fiber, single radial/azimuthal mode transmission, elliptical-hole lattice, finite element method.

\section{INTRODUCTION}

Single mode fibers (SMFs) play an important role for mode multiplexing in optical communication systems in recent years. SMFs can be classified into several types by the guidance mechanisms, such as conventional SMFs based on the total internal reflection [1], Bragg fibers based on the effect of Bragg reflection [2]- [4], and photonic crystal fibers (PCFs) based on the concept of photonic crystals [5], [6]. Due to the flexible structures and unique properties of PCFs, a lot of attentions have been paid on their research and development. One of the important parameters of PCFs is the birefringence, and there are two main methods to achieve a high birefringence, One is by introducing elliptical holes or other asymmetric air holes into the core [7]- [10], since elliptical holes are effective to break the symmetry [8]. The other is to change the refractive index contrast between the core and cladding by doping a liquid crystal in the core [12] or utilizing other materials such as metal, polymer, plastic, and so on. Due to the high birefringence, lots of single-polarization (single $x$ - or $y$ polarization) single-mode (single $\mathrm{HE}_{11}$ mode) fibers have been demonstrated earlier. However, a single radial or azimuthal mode fiber has never been investigated by the PCFs so far.

In this letter, to our knowledge, we firstly propose the novel single radial/azimuthal mode PCFs, which have an anisotropic elliptical-hole lattice core (EHLC). First, we put forward an analysis to confirm that how much of the birefringence can

Z. Zhong and Y. Tsuji are with the Division of Information and Electronic Engineering, Muroran Institute of Technology, Muroran 050-8585, Japan (email:15043030,y-tsuji@mmm.muroran-it.ac.jp).

M. Eguchi is with the Department of Opto-Electronic System Engineering, Chitose Institute of Science and Technology, Chitose 066-8655, Japan (email:megu@ieee.org).

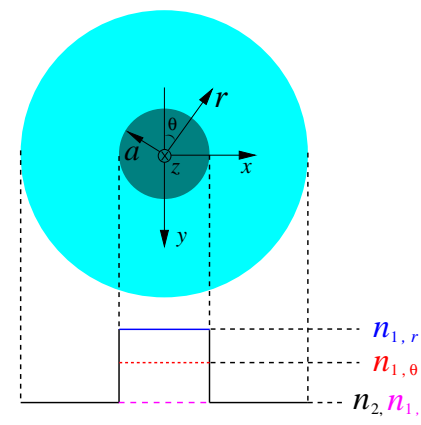

\begin{tabular}{l} 
Parameters \\
\hline$a:$ radius \\
\hline$n_{0}=1.444$ \\
\hline$n_{1, z}=n_{0}$ (longitudinal) \\
$n_{1, r}=n_{0}+\frac{B}{2}$ (radial) \\
$n_{1, \theta}=n_{0}-\frac{B}{2}$ (azimuthal) \\
\hline$n_{2}=n_{0}$ (isotropic) \\
\hline$B: 0.02 \sim 0.10$ \\
\hline
\end{tabular}

Fig. 1. The schematic and parameters of the equivalent SI-fiber model. The core and cladding are characterized by anisotropic and isotropic materials, respectively. The refractive indices along different directions in the core are assumed in the cylindrical coordinate system. $B\left(=n_{1, r}-n_{1, \theta}\right)$ represents the birefringence, defined as the refractive index difference between the radial and azimuthal directions of the core.

support the single radial mode transmission based on an equivalent step index- (SI-) fiber model in Section II. Next, we investigate the performances of the single radial mode PCF and single azimuthal mode PCF by employing the full vectorial finite element method (FV-FEM) [13] in Sections III and IV, respectively. Finally, we give a brief conclusion of all analyses in Section V.

\section{EQuivalent STEP INDEX-FIBER MODEL}

For convenience, we consider an equivalent model based on a 2-layer SI-fiber with a radius $a$ of the core, which is shown in Fig. 1. In order to earn a birefringence $B$, we assume an anisotropic material in the core, whose refractive indices along longitudinal, radial, and azimuthal directions are defined as $n_{1, z}=n_{0}, n_{1, r}=n_{0}+\frac{B}{2}$, and $n_{1, \theta}=n_{0}-\frac{B}{2}$, respectively. The cladding is characterized by an isotropic material, whose refractive index is defined as $n_{2}=n_{0}$, where $n_{0}$ is assumed to be 1.444. At first, we discuss the dispersion of $\Delta n_{\text {eff }}$ as a function of core radius $a$. Considering a high birefringence of 0.08 obtained in elliptical-hole holey fiber [14], $B$ is changed from 0.02 to 0.10 with an interval of 0.02 and the operating wavelength is set to $\lambda=1.55 \mu \mathrm{m}$. $\Delta n_{\text {eff }}$ stands for the difference of the modal effective refractive indices between the radial and $\mathrm{HE}_{11}$ modes, that can be expressed as the following equation:

$$
\Delta n_{\mathrm{eff}}=n_{\mathrm{eff}}^{\mathrm{Radial}}-n_{\mathrm{eff}}^{\mathrm{HE}_{11}},
$$

where $n_{\text {eff }}^{\text {Radial }}$ and $n_{\text {eff }}^{\mathrm{HE}_{11}}$ indicate the modal effective refractive indices of radial and $\mathrm{HE}_{11}$ modes, respectively. It can be observed in Fig. 2 that, $\Delta n_{\text {eff }}$ reaches to the maximum of $2.647 \times 10^{-3}$ under the conditions of $a=3.5 \mu \mathrm{m}$ and 


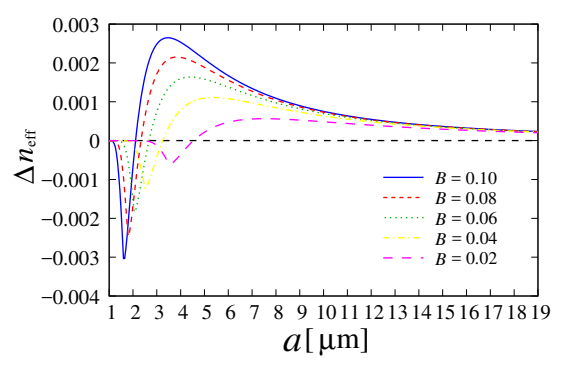

Fig. 2. The difference of the modal effective refractive indices between radial and $\mathrm{HE}_{11}$ modes as a function of core radius $a$, under the operation wavelength $\lambda=1.55 \mu \mathrm{m}$.

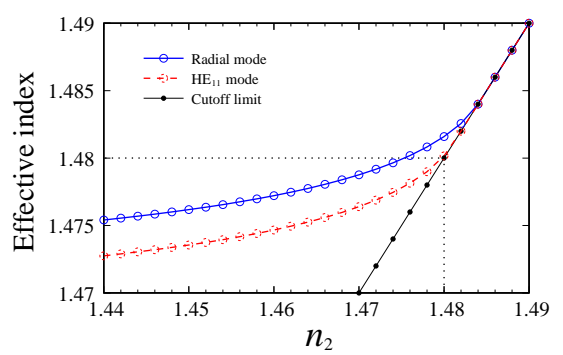

Fig. 3. The modal effective indices of radial and $\mathrm{HE}_{11}$ modes with respect to the refractive index of cladding $n_{2}$.

$B=0.10$. Moreover, $\Delta n_{\text {eff }}$ decreases with an increase in the core radius. In order to achieve the single radial mode transmission, we need to adjust the refractive index of cladding. Fig. 3 shows the modal effective indices of radial and $\mathrm{HE}_{11}$ modes with respect to the refractive index of cladding $n_{2}$. When the effective index equals the cladding index, the mode is in cutoff state, and thus we refer to the line with black dots corresponding to the cladding index as cutoff limit. The line with blue-colored open circles and the line with red-colored dashed circles indicate the modal effective indices of radial and $\mathrm{HE}_{11}$ modes, respectively. We can observe that $\mathrm{HE}_{11}$ mode crosses with the cutoff limit first at $n_{2}=1.480$, and then the radial mode crosses at $n_{2}=1.484$. Therefore, at the refractive index range from 1.480 to 1.484 , the single radial mode transmission can be achieved in the equivalent SI-fiber. The magnetic field distributions and Poynting vectors of radial and $\mathrm{HE}_{11}$ modes for $n_{2}=1.482$ are shown in Fig. 4. It can be observed that, the $\mathrm{HE}_{11}$ mode is not guided and only the radial mode is confined in the core. That means that the single mode transmission can be achieved by the anisotropy between the radial and azimuthal directions of the core in the equivalent SI-fiber. Furthermore, The modal effective indices of radial and $\mathrm{HE}_{11}$ modes and the cladding index as a function of wavelength is shown in Fig. 5. The light blue shaded region represents the non-confinement region, where the modal effective indices of radial (blue line) and $\mathrm{HE}_{11}$ modes (red dashed line) are lower than that of the cladding index (dot line). In the wavelength range from 1.48 to $1.68 \mu \mathrm{m}$, the single radial mode transmission is achieved in the equivalent SI-fiber.

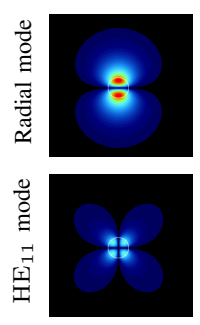

$H_{x}$
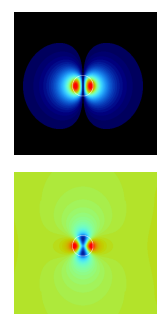

$H_{y}$

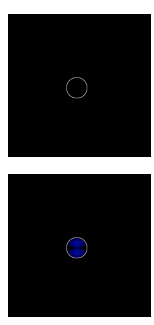

$H_{z}$

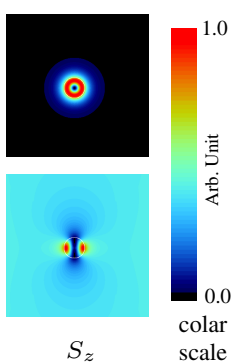

Fig. 4. The magnetic field distributions $\left(H_{x}, H_{y}, H_{z}\right)$ and Poynting vectors $\left(S_{z}\right)$ of radial and $\mathrm{HE}_{11}$ modes, at $\lambda=1.55 \mu \mathrm{m}$ and $n_{2}=1.482$.

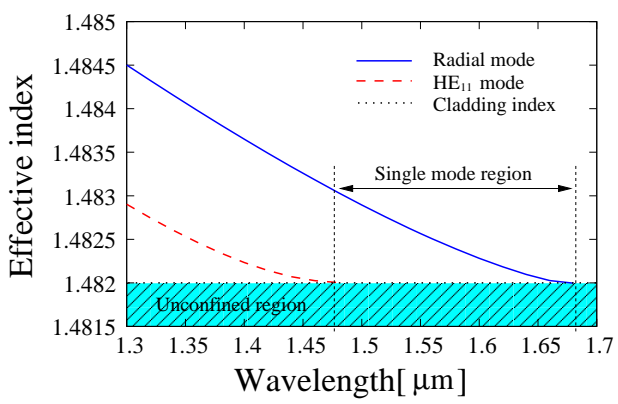

Fig. 5. The modal effective indices of radial and $\mathrm{HE}_{11}$ modes and the cladding index as a function of wavelength.

\section{Single RAdiAL MOdE PHOTONIC CRYSTAL FIBER}

On the basis of the equivalent SI-fiber model described in Section II, we propose a novel single radial mode PCF with an actinomorphic EHLC to achieve the single radial mode transmission. Fig. 6 shows the schematic of the proposed PCF and the rough mesh division of a quarter cross-section. As shown in Fig. 6 (a), the proposed PCF is characterized by the fused-silica $\left(\mathrm{SiO}_{2}\right)$ with defected air holes in a triangular lattice. The centers of all air holes are arranged by the lattice pitch of $\Lambda$. The core is arranged by elliptical holes with the radii $R_{L}$ and $R_{S}$ for the major and minor axes, respectively. With these major axes of elliptical holes along the radial directions and a large circular hole with a radius of $R_{a}$ in the center, the radial mode will be guided more easily than the $\mathrm{HE}_{11}$ mode. The radius of cladding air hole is represented by $R_{c}$. Considering the material dispersion of $\mathrm{SiO}_{2}$ existing in the real applications, we calculate the refractive index of fused-silica based on the Sellmeier equation [15], which is expressed as follows:

$$
\begin{aligned}
n^{2}(\lambda)=1+\frac{0.6961663 \lambda^{2}}{\lambda^{2}-(0.0684043)^{2}} & +\frac{0.4079426 \lambda^{2}}{\lambda^{2}-(0.1162414)^{2}} \\
& +\frac{0.8974794 \lambda^{2}}{\lambda^{2}-(9.8961610)^{2}} .
\end{aligned}
$$

The refractive index of air is set to 1.0. Due to the structural symmetries along the $x$ - and $y$-axes, we can only calculate a quarter cross-section to reduce the analysis cost. In Fig. 6 (b), a rough mesh division of a quarter cross-section of the PCF is shown, where the symmetric boundary conditions $\left(\Gamma_{1}, \Gamma_{2}\right)$ and $\left(\Gamma_{3}\right.$ and $\left.\Gamma_{4}\right)$ are loaded by the perfect magnetic conductor (PMC) and the perfect electric conductor (PEC),respectively, for the single radial mode PCF analyses. For more accurate 


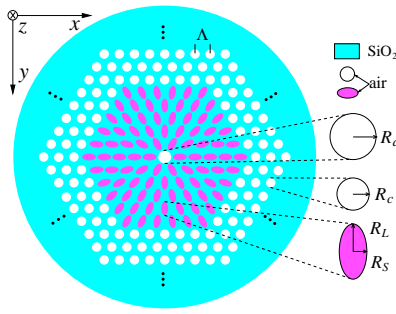

(a)

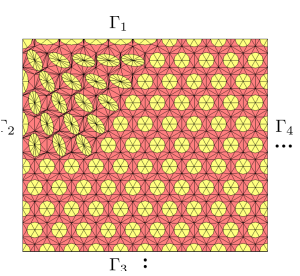

(b)
Fig. 6. The schematic of the proposed single radial mode PCF with an actinomorphic EHLC (a), and the rough mesh division of a quarter crosssection (b), where the symmetric boundaries $\left(\Gamma_{1}, \Gamma_{2}\right)$ and $\left(\Gamma_{3}\right.$ and $\left.\Gamma_{4}\right)$ are loaded by PMC and PEC, respectively.

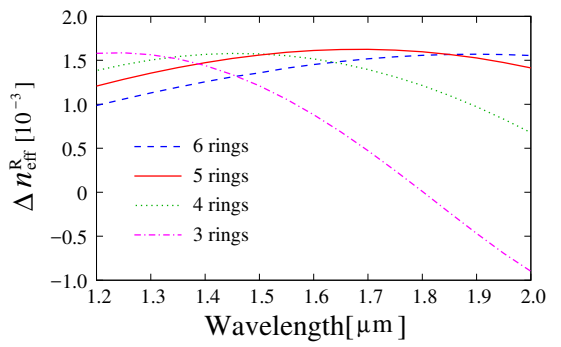

Fig. 7. The modal effective index difference $\Delta n_{\text {eff }}^{\mathrm{R}}$ in different core rings as a function of wavelength.

analyses, we give the rough quarter mesh another 50,000 divisions. After that, we select $\Lambda=1 \mu \mathrm{m}, R_{a}=0.40 \Lambda$, $R_{S}=0.24 \Lambda$, and $R_{L}=2 R_{S}$ as the optimizing parameters to earn the highest birefringence. First, we set $R_{c}=\sqrt{2 R_{S}^{2}}$, which makes both areas of an elliptical hole and an air hole in the cladding equal. In other words, we approximate that the modal effective indices of core and cladding are the same to guide the radial and $\mathrm{HE}_{11}$ modes, simultaneously. Fig. 7 shows the relation between the modal effective index difference $\Delta n_{\mathrm{eff}}^{\mathrm{R}}\left(=n_{\mathrm{eff}}^{\mathrm{Radial}}-n_{\mathrm{eff}}^{\mathrm{HE}_{11}}\right)$ and the number of core rings with respect to wavelength. At the communication wavelength of $1.55 \mu \mathrm{m}$, the maximum of $\Delta n_{\mathrm{eff}}^{\mathrm{R}}$ reaches $1.591 \times 10^{-3}$ with a 5-ring core. Next, we select an optimal size of $R_{c}$ to achieve the single radial mode transmission in the proposed PCF. when $R_{c}=0.322 \Lambda$, the bandwidth of single radial mode transmission is the widest. In the wavelength range from 1.45 to $1.66 \mu \mathrm{m}$ (a bandwidth of $210 \mathrm{~nm}$ ), the single radial mode transmission is achieved in the proposed PCF with an actinomorphic EHLC. At that time, the magnetic field distributions of both modes are shown in Fig. 8 at a wavelength of $1.55 \mu \mathrm{m}$. The single radial mode is confined, while the $\mathrm{HE}_{11}$ mode is not guided. Table I represents the single mode bandwidth (SMB) and its wavelength range (WR) of the proposed PCF, when the elliptical and cladding holes are deviated within $\pm 0.3 \%$. when elliptical holes become large (represented by "+"), the single radial mode band shifts toward longer wavelength and the bandwidth is slightly expanded. When they become small (represented by "-"), the band shifts toward to shorter wavelength. On the contrary, the circumstance of cladding holes represents the opposite trend. All the analyses are employed by the FV-FEM with curvilinear hybrid edge/nodal elements, the analyzed region is

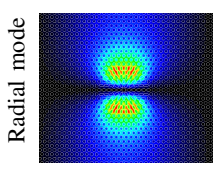

$H_{x}$

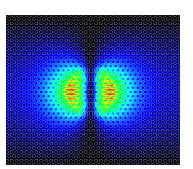

$H_{y}$

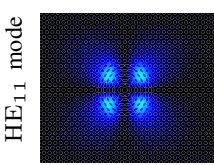

$H_{x}$

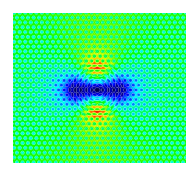

$H_{y}$
Fig. 8. The magnetic field distributions $\left(H_{x}\right.$ and $\left.H_{y}\right)$ of both modes in the proposed single radial mode PCF, at $\lambda=1.55 \mu \mathrm{m}$.

TABLE I

SINGLE RADIAL MODE BANDWIDTH AND ITS WAVELENGTH RANGE VERSUS THE DEVIATION LEVEL OF ELLIPTICAL AND CLADDING HOLES

\begin{tabular}{ccccc}
\hline \hline \multirow{2}{*}{$\begin{array}{c}\text { Deviation } \\
\text { level }\end{array}$} & \multicolumn{2}{c}{ Elliptical holes } & \multicolumn{2}{c}{ Cladding holes } \\
\cline { 2 - 5 } & SMB [nm] & WR $[\mu \mathrm{m}]$ & SMB $[\mathrm{nm}]$ & WR $[\mu \mathrm{m}]$ \\
\hline$-0.3 \%$ & 171 & $1.619 \sim 1.790$ & 225 & $1.262 \sim 1.487$ \\
$-0.2 \%$ & 182 & $1.565 \sim 1.747$ & 218 & $1.329 \sim 1.547$ \\
$-0.1 \%$ & 193 & $1.510 \sim 1.703$ & 212 & $1.392 \sim 1.604$ \\
$0 \%$ & 210 & $1.450 \sim 1.660$ & 210 & $1.450 \sim 1.660$ \\
$+0.1 \%$ & 209 & $1.400 \sim 1.609$ & 191 & $1.516 \sim 1.707$ \\
$+0.2 \%$ & 218 & $1.340 \sim 1.558$ & 175 & $1.580 \sim 1.755$ \\
$+0.3 \%$ & 223 & $1.282 \sim 1.505$ & 163 & $1.638 \sim 1.801$ \\
\hline \hline
\end{tabular}

about $18.0 \times 15.6 \mu \mathrm{m}^{2}$, and the total unknowns are 295,550 in the single radial mode PCF analyses.

\section{Single aZimuthal Mode Photonic CRYSTAL Fiber}

Based on the discussions in Sections II and III, we consider the schematic of single azimuthal mode PCF with a multiannulus EHLC as shown in Fig. 9, which is formed by rotating the elliptical holes in the core of Fig. 6 by $90^{\circ}$. As same as the circumstance in the single radial mode PCF analyses, only a quarter cross-section is calculated, and symmetric boundary conditions $\left(\Gamma_{1}^{\prime}, \Gamma_{2}^{\prime}\right)$ and $\left(\Gamma_{3}^{\prime}, \Gamma_{4}^{\prime}\right)$ are loaded by PEC and PMC, respectively, which is shown in Fig. 9 (b). After rotating, the $\mathrm{SiO}_{2}$ area around the large circular hole gets increased. Fig. 10 shows the modal effective indices of two modes and its difference $\Delta n_{\text {eff }}^{\mathrm{A}}\left(=n_{\mathrm{eff}}^{\mathrm{Azimuthal}}-n_{\mathrm{eff}}^{\mathrm{HE}_{11}}\right)$ as functions of the radius of large circular hole $R_{a}$. Here the radius of cladding air hole is set to $R_{c}=\sqrt{R_{S}^{2}}$. It can be observed that, with the increasing $R_{a}$, the effective index of azimuthal mode is almost flat, conversely, the effective index of $\mathrm{HE}_{11}$ mode declines. When $R_{a}=0.49 \Lambda, \Delta n_{\mathrm{eff}}^{\mathrm{A}}$ reaches the maximum of $1.205 \times 10^{-3}$. On the condition, when we select $R_{c}=0.3235 \Lambda$, the single azimuthal mode transmission is achieved in the wavelength range from 1.50 to $1.60 \mu \mathrm{m}$, and the bandwidth of that is $100 \mathrm{~nm}$. Fig. 11 shows the magnetic field distributions of both modes at a wavelength of $1.55 \mu \mathrm{m}$. We can observe that the single azimuthal mode has an orthogonal relation with the single radial mode. And the FEM computational window is set to be the same as that in the single radial mode PCF analyses in the previous Section, except that the total unknowns are 295,854 in the single azimuthal mode PCF analyses. Table II represents the single azimuthal mode bandwidth and its wavelength range of the proposed PCF, when the elliptical and cladding holes are deviated only in $\pm 0.1 \%$. The phenomenon of the band shift is as same as that in the single radial mode PCF. With a minor change of the air holes, the single azimuthal mode transmission is hardly achieved in the wavelength of $1.55 \mu \mathrm{m}$ in 


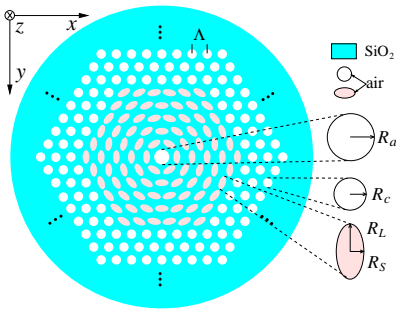

(a)

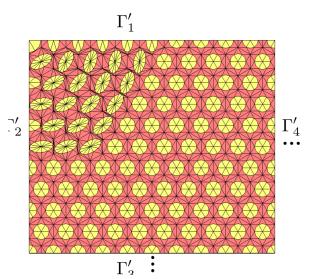

(b)
Fig. 9. The schematic of the proposed single azimuthal mode PCF with a multi-annulus EHLC (a), and the rough mesh division of a quarter crosssection (b), where the symmetric boundaries $\left(\Gamma_{1}^{\prime}, \Gamma_{2}^{\prime}\right)$ and $\left(\Gamma_{3}^{\prime}\right.$ and $\left.\Gamma_{4}^{\prime}\right)$ are loaded by PEC and PMC, respectively.

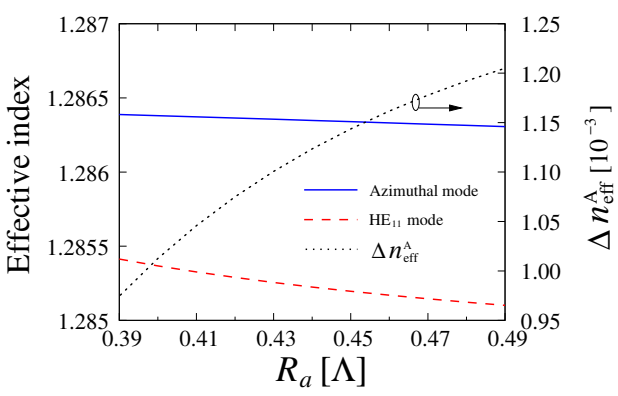

Fig. 10. The modal effective indices of two modes and its difference $\Delta n_{\text {eff }}^{\mathrm{A}}$ as functions of the radius of large circular hole $R_{a}$ with the radius of cladding air hole $R_{c}=\sqrt{R_{S}^{2}}$.

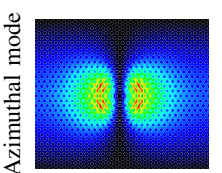

$H_{x}$

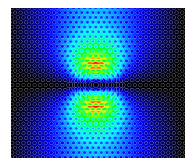

$H_{y}$

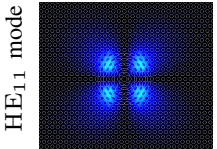

$H_{x}$

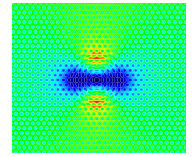

$H_{y}$
Fig. 11. The magnetic field distributions $\left(H_{x}\right.$ and $\left.H_{y}\right)$ of both modes in the proposed single azimuthal mode PCF, at $\lambda=1.55 \mu \mathrm{m}$.

the proposed PCF with a multi-annulus EHLC. The presented results have been estimated for uniform structural fluctuations. So, the presented tolerances are the worst estimations. In fact, the random structural fluctuations may be somewhat canceled out each other for the wavelength range deviations.

\section{CONCLUSION}

The single radial/azimuthal mode transmission has been numerically demonstrated by the proposed PCFs with an anisotropic EHLC. The analyses based on the equivalent SIfiber model give the theoretical criteria for designing the proposed PCFs. The bandwidths of $210 \mathrm{~nm}$ for the single radial mode transmission and $100 \mathrm{~nm}$ for the single azimuthal mode transmission have been achieved in the PCFs with an actinomorphic EHLC and with a multi-annulus EHLC, respectively. Although, the precision problem in fabricating elliptical holes does exist, the research and development of the fabrication techniques are in high progress. Combined with the existing techniques such as the stack and draw technique [16], post-processing technique, flame brushing technique, interlaced stacking technique and so on [17], the fabrication of elliptical holes will not be the most critical problem in the applications. Although the operating wavelength of the
TABLE II

SINGLE AZIMUTHAL MODE BANDWIDTH AND ITS WAVELENGTH RANGE VERSUS THE DEVIATION LEVEL OF ELLIPTICAL AND CLADDING HOLES

\begin{tabular}{ccccc}
\hline \hline \multirow{2}{*}{$\begin{array}{c}\text { Deviation } \\
\text { level }\end{array}$} & \multicolumn{2}{c}{ Elliptical holes } & \multicolumn{2}{c}{ Cladding holes } \\
\cline { 2 - 5 } & SMB $[\mathrm{nm}]$ & WR $[\mu \mathrm{m}]$ & SMB [nm] & WR $[\mu \mathrm{m}]$ \\
\hline$-0.1 \%$ & 78 & $1.563 \sim 1.641$ & 106 & $1.420 \sim 1.526$ \\
$0 \%$ & 100 & $1.500 \sim 1.600$ & 100 & $1.500 \sim 1.600$ \\
$0.1 \%$ & 100 & $1.430 \sim 1.530$ & 75 & $1.570 \sim 1.645$ \\
\hline \hline
\end{tabular}

EHLC proposed here is sensitive to structural deviation, we would like to discuss more insensitive structures in the future work. Based on this, we believe that the proposed PCFs will be a significant advance for the mode multiplexing in optical communication systems.

\section{REFERENCES}

[1] T. Okoshi, "Single-polarization single-mode optical fibers," IEEE $J$. Quantum Electron., vol. QE-17, no. 6, pp. 879-884, Jun. 1981.

[2] P. Yeh, A. Yariv, and E. Marom, "Theory of Bragg fiber," J. Opt. Soc. Am., vol. 68, no. 9, pp. 1196-1201, Sep. 1978.

[3] A. Argyros, I. M. Bassett, M. A. V. Eijkelenborg, and M. C. J. Large, "Analysis of ring-structured Bragg fibers for single TE mode guidance," Opt. Express, vol. 12, no. 15, pp. 2688-2698, Jun. 2004.

[4] D. Mao, Z. Ouyang, J. C. Wang, and C. P. Liu, "Single-TM-mode Bragg fibers made of magnetic materials," Opt. Express, vol. 16, no. 2, pp. 628-635, Jan. 2008.

[5] T. A. Birks, J. C. Knight, and P. St. J. Russell, "Endlessly single-mode photonic crystal fiber," Opt. Lett., vol. 22, no. 13, pp. 961-963, Jul. 1997.

[6] P. St. J. Russell, "Photonic-crystal fibers," J. Lightw. Technol., vol. 24, no. 12, pp. 4729-4749, Dec. 2006.

[7] K. Saitoh and M. Koshiba, "Single-polarization single-mode photonic crystal fibers," IEEE Photon. Technol. Lett., vol. 15, no. 10, pp. 13841386, Oct. 2003.

[8] M. Eguchi and Y. Tsuji, "Single-mode single-polarization holey fiber using anisotropic fundamental space-filling mode," Opt. Lett., vol. 32, no. 15, pp. 2112-2114, Aug. 2007.

[9] K. Ichikawa, Z. Zhang, Y. Tsuji, and M. Eguchi, "A single-polarization holey fiber with anisotropic lattice of circular air holes," J. Lightw. Technol., vol. 33, no. 18, pp. 3866-3871, Sep. 2015.

[10] L. An, Z. Zheng, Z. Li, T. Zhou, and J. Cheng, "Ultrahigh birefringent photonic crystal fiber with ultralow confinement loss using four airholes in the core," J. Lightw. Technol., vol. 27, no. 15, pp. 3175-3180, Aug. 2009.

[11] S. E. Kim, B. H. Kim, C. G. Lee, S. Lee, K. Oh, and C. S. Kee, "Elliptical defected core photonic crystal fiber with high birefringence and negative flattened dispersion," Opt. Express, vol. 20, no. 2, pp. 1385 1391, Jan. 2012.

[12] M. F. O. Hameed and S. S. A. Obayya, "Modal analysis of a novel soft glass photonic crystal fiber with liquid crystal core," J. Lightw. Technol., vol. 30, no. 1, pp. 96-102, Jan. 2012.

[13] M. Koshiba and Y. Tsuji, "Curvilinear hybrid edge/nodal elements with triangular shape for guided-wave problems," J. Lightw. Technol., vol. 18, no. 4, pp. 737-743, May 2000.

[14] M. Eguchi and Y. Tsuji, "Squeezed lattice elliptical-hole holey fiber with extremely high birefringence," Opt. Lett., vol. 33, no. 16, pp. 1792-1794, Aug. 2008.

[15] N. Karasawa, S. Nakamura, N. Nakagawa, M. Shibata, R. Morita, H. Shigekawa, and M. Yamashita, "Comparison between theory and experiment of nonlinear propagation for a-few-cycle and ultrabroadband optical pulses in a fused-silica fiber," IEEE. J. Quantum Electron., vol. 37, no. 3, pp. 398-404, Mar. 2001.

[16] D. Pysz, I. Kujawa, R. Stepien, M. Klimczak, A. Filipkowski, M. Franczyk, L. Kociszewski, J. Buzniak, K. Harasny, and R. Buczynski, "Stack and draw fabrication of soft glass microstructured fiber optics," Bull. Pol. Acad. Sci. Tech. Sci., vol. 62, no. 4, pp. 667-682, Dec. 2014.

[17] E. C. Mägi, P. Steinvurzel, and B. J. Eggleton, "Tapered photonic crystal fibers," Opt. Exp., vol. 12, no. 5, pp. 776-784, Mar. 2004. 